\title{
Remarques sur la revision de l'article 30 de la Convention de Genève
}

\author{
Schindler, Dietrich
}

\begin{abstract}
En vue des travaux à entreprendre pour la revision de la Convention de Genève de 1929, le Comité international de la Croix-Rouge a tenu à connaître l'avis de juristes qualifiés du droit international sur la réforme de l'article 30. Il s'est adressé à cet effet à M. A. Hammarskjöld, juge à la Cour permanente de justice internationale, et à M. Dietrich Schindler, professeur de droit à l'Université de Zurich
\end{abstract}

DOI: https://doi.org/10.1017/s1026881200101382

Posted at the Zurich Open Repository and Archive, University of Zurich

ZORA URL: https://doi.org/10.5167/uzh-154022

Journal Article

Published Version

Originally published at:

Schindler, Dietrich (1937). Remarques sur la revision de l'article 30 de la Convention de Genève. International Review of the Red Cross, 19(221):510-519.

DOI: https://doi.org/10.1017/s1026881200101382 


\section{Dietrich SCHINDLER,}

professeur de droit à l'Université de Zurich.

\section{Remarques sur la revision de l'article 30 de la Convention de Genève.}

En vue des travaux à entreprendre pour la revision de la Convention de Genève de 1929, le Comité international de la Croix-Rouge a tenu à connaître l'avis de juristes qualifiés du droit international sur la réforme de l'article 30.

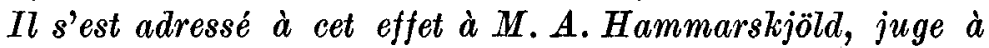
la Cour permanente de justice internationale, et $\grave{a}$ M. Dietrich Schindler, professeur de droit à l'Université de Zurich.

Le texte de M. Hammarskjöld parâ̂tra dans la Zeitschrift für aussländisches öffentliches Recht und Völkerrecht. on trouvera ci-dessous celui au professeur Schindler. (N.d.l.R.).

La question de la procédure à suivre en cas de violation de la Convention a été longtemps discutée à la Conférence de 1929. Aucune des propositions visant à une réglementation précise n'a recueilli l'adhésion de tous les Etats. C'est pourquoi on s'est contenté d'introduire un article qui, tout en stipulant l'obligation d'ouvrir une enquête à la ḋemande d'un belligérant, laisse le soin d'en fixer la procédure aux parties intéressées.

La modification de l'article 30 doit porter sur la procédure d'enquête ; en effet, ce qui importe surtout, c'est de trouver un moyen de déterminer aussi rapidement que possible - et de manière impartiale - l'exactitude des faits allégués par une des Parties en cause et considérés par elle comme une violation de la Convention.

Il serait désirable d'instituer une juridiction, appelée à trancher obligatoirement les conflits issus d'une telle 


\section{Revision de l'article 30 \\ de la Convention de Genève.}

violation. La création de cette juridiction permettrait à la fois de constater les faits et de trancher des questions de droit. Cependant il parait peu problable $q u$ 'une clause y relative puisse être insérée dans la Convention. Pour assurer l'exécution de la Convention il est peut-être plus important d'instituer une procédure rapide d'enquête qu'une juridiction. L'objet de cette étude concerne exclusivement la procédure destinée à constater les infractions à la Convention. Les Etats qui désireraient établir une juridiction auront facilement accès à la Cour permanente de justice internationale.

On pourrait envisager de confier l'enquête à une des Commissions permanentes, qui ont été créées en application des traités d'enquête ou de conciliation. Certes, en cas dé guerre, un traité de ce genre doit être considéré comme suspendu ou annulé; par conséquent, il ne pourrait être appliqué tel quel. De plus, la tâche d'une commission d'enquête ne serait pas la même que celle de la plupart des commissions permanentes actuelles. Mais cela n'empêche pas les parties de recourir, pour cette enquête, aux services des personnes qu'elles avaient désignées comme membres de la Commission permanente, instituée entre elles. Cependant toutes ces personnes ne sauraient conduire l'enquête comme il convient. En effet, les ressortissants de nations en guerre, membres de la commission ne peuvent pas se rendre en territoire ennemi ; seuls les membres désignés d'un commun accord pourraient se charger des investigations dans les deux pays belligérants. Même ces enquêteurs peuvent appartenir à des nations belligérantes, au cas où la guerre sévit dans plus de deux Etats, de sorte que la commission pourrait être dans l'impossibilité de rendre les services qu'on lui demande. Il est donc exclu d'insérer dans la Convention de Genève une clause par laquelle les belligérants devraient s'en remettre, pour les enquêtes relatives aux violations de la Convention, à une des commissions 


\section{Dietrich Schindler.}

permanentes actuelles. C'est seulement dans certains cas - qui ne peuvent être réglés à l'avance - que l'appel à une commission existante peut être utile.

Cependant une solution paraît possible : c'est la création par les deux belligérants, dès le début des hostilités, d'une commission d'enquête. Mais constituer à l'avance une commission, c'est prévoir des violations, ce qui, du point de vue psychologique, n'est peut-être pas très heureux. De plus, il sera peut-être difficile de s'entendre entre belligérants sur la composition d'un tel organe. Enfin, une autre objection s'impose encore : s'il y a plusieurs belligérants, on devrait créer un grand nombre de commissions dont la plupart n'auraient probablement aucun emploi. Par conséquent, obliger formellement les Etats à constituer des commissions d'enquête, dès le début des hostilités, ne paraît pas une solution pratique du problème. Mais rien ne s'oppose à ce que la Convention de Genève ne permette aux belligérants de constituer entre eux de telles commissions. Du reste, le texte actuel de l'article 30 ne s'y oppose pas.

Pour améliorer l'article 30 , il faudrait instituer une procédure qui permettrait la formation rapide d'une commission d'enquête à la requête d'un des belligérants. Pour y arriver, il me paraît indispensable de faire intervenir dans cette procédure une instance neutre et indépendante des belligérants. Mais laquelle choisir ?

Dans cet ordre d'idées, il faut relever que l'Union interparlementaire, à sa session de 1913, vota la résolution suivante : "Considérant que de nombreuses infractions à la Convention de la Haye du 18 octobre 1907 concernant les lois et coutumes de la guerre ont été dénoncées de part et d'autre..., considérant qu'il doit être dans l'intérêt d'un belligérant que de semblables allégations soient examinées par une instance impartiale et au moment opportun, considérant l'importance de créer, pour les violations éventuelles du droit, des sanctions 


\section{Revision de l'article 30 de la Convention de Genève.}

efficaces, la XVIII Conférence interparlementaire émet le vœu que ces questions soient mises à l'ordre du jour de la prochaine Conférence de la paix."

En vue de la Conférence interparlementaire qui devait se tenir à Stockholm, en 1914, si la guerre mondiale n'avait pas éclaté, la résolution suivante avait été élaborée par le groupe russe :

“1. Qu'en cas de guerre entre les Puissances signataires... le Conseil administratif de la Cour permanente d'arbitrage de la Haye soit chargé de nommer des Commissions internationales de contrôle et d'enquête auprès des belligérants. 2. Que les dites Commissions soient composées de membres choisis par le Conseil parmi les ressor. tissants des Etats neutres, jouissant de la plus haute considération morale et d'une compétence reconnue dans les questions de droit international de la guerre, au nombre de trois au moins pour chaque Commission... $\nu^{1}$ ).

L'idée de faire nommer des commissions d'enquête par un organe international paraît heureuse. Au contraire, la constitution d'une commission internationale auprès de chaque Etat en guerre ne paraît pas toujours réalisable; les Etats considéreraient facilement ce contrôle permanent comme une marque de défiance à leur égard. Cette difficulté n'existerait peut-être pas si la commission de contrôle était composée, par exemple, des agents diplomatiques de puissances neutres accréditées auprès du belligérant. Cette " commission " ne serait pas nommée par un organe international, elle se constituerait ellemême. Evidemment l'ensemble des agents diplomatiques, c'est-à-dire le corps diplomatique, ne pourra pas procéder lui-même à des enquêtes. Mais il pourrait - et cela dès le début des hostilités - nommer une commission,

1 Efremoff, dans Recueil des cours de l'Académie de droit international, 1927, III p. 22/3.

Il convient de rappeler que la Dotation Carnegie pour la paix internationale avait envoyé une commission d'enquête sur le théâtre de la guerre balkanique. EFrEmofF, loc. eit. 


\section{Dietrich Schindler.}

composée de ressortissants neutres, choisis de préférence parmi le personnel diplomatique ; elle ouvrirait l'enquête dès qu'une plainte serait déposée contre un Etat en cause. De telles réclamations seraient portées à la connaissance $d u$ corps diplomatique par l'entremise de la puissance protectrice. Des, commissions de ce genre ont l'avantage de pouvoir entrer en action très rapidement. Il est moins certain qu'elles offrent toutes les garanties voulues d'impartialité. Du reste, il ne s'agit pas seulement de faire une enquête contre l'Etat accusé, mais aussi d'examiner si les réclamations de l'Etat plaignant sont fondées. A cette fin, ces commissions ne peurent pas fonctionner, à moins qu'on ne veuille donner à la commission constituée dans l'Etat adverse le droit d'examiner préalablement toute plainte. Un système de ce genre a été proposé à la Conférence du désarmement, mais il est très douteux qu'il puisse fonctionner sans l'existence d'un organe central (cet organe eût été la Commission permanente $d u$ désarmement). Le Comité international de la Croix-Rouge pourrait-il être cet organe central ? La réponse à cette question devrait être laissée au Comité lui-même.

Somme toute, une commission constituée entre deux ou plusieurs Etats paraît préférable à des commissions rattachées chacune à un Etat. Seulement, la nomination d'une telle commission ne peut être laissée aux belligérants. Il importe donc de trouver une instance internationale qui serait à même, soit de nommer ces commissions, soit de faire elle-même les enquêtes.

Le Conseil d'administration de la Cour permanente d'arbitrage ne paraît pas être l'organe indiqué pour cette tâche. La Cour permanente de justice internationale pourrait certainement faire des enquêtes ou nommer des commissions, cependant il est peu probable que tous les Etats signataires de la Convention de Genève soient disposés à reconnaître sa compétence en cette matière. 


\section{Revision de l'article 30 de la Convention de Genève.}

En outre, si le théâtre de la guerre se trouve dans un autre continent, l'enquête ouverte par la Cour risquerait d'être entreprise trop tard.

Le Conseil de la Société des Nations ne peut remplir cette fonction. Par la tâche qui lui incombe en vertu du Pacte de la Société des Nations (article 16) il ne pourra pas toujours avoir l'impartialité indispensable.

L'instance internationale la plus indiquée pour remplir cette tâche est le Comité international de la Croix-Rouge. Il peut faire des enquêtes lui-même ou nommer des commissions composées de personnalités qualifiées. Son impartialité absolue et ses expériences en la matière le désignent tout particulièrement pour cette fonction. Si les deux Etats font appel au Comité international de la Croix-Rouge, il peut nommer une commission sans compromettre en quoi que ce soit sa neutralité.

Cependant il serait difficile d'inscrire dans une Convention universelle l'obligation de faire nommer des commissions d'enquête par le Comité international de la CroixRouge. La difficulté qui vient d'être mentionnée surgit de nouveau ici : s'il y a une guerre entre deux pays éloignés, il serait probablement difficile pour le Comité international de la Croix-Rouge de constituer des commissions aptes à procéder rapidement à l'enquête. Une commission, formée de personnalités habitant l'Europe, arriverait trop tard. C'est ainsi qu'à l'occasion du conflit sino-japonais la nomination d'une commission d'étude avait été décidée par le Conseil de la Société des Nations, le 10 décembre 1931 ; mais cette commission - la Commission Lytton - n'est arrivée au Japon que le 29 février 1932. Cependant une autre commission constituée à Shanghaï par les représentants officiels des membres du Conseil de la Société des Nations a été à même de donner ses premiers rapports au bout de quelques jours. Par conséquent, la Convention de Genève devrait prévoir une autre procédure pour la nomination des 


\section{Dietrich Schindler.}

commissions d'enquête. Dans cette autre procédure, un rôle important serait confié aux seules instances qui entrent encore en ligne de compte : les puissances protectrices.

La Convention relative au traitement des prisonniers de guerre charge les puissances protectrices d'une fonction importante dans "l'organisation du contrôle " de l'application de la Convention. Ces dispositions ne pourraient pas être appliquées telles quelles aux enquêtes ouvertes en cas de violations alléguées de la Convention de Genève; en effet, ces enquêtes, contrairement à celles qui sont prévues pour le contrôle des camps de prisonniers de guerre, ne devraient se faire qu'exceptionnellement, mais alors d'une façon très rapide. Cependant, dans l'un et dans l'autre des cas, les puissances protectrices sont destinées à exercer une fonction importante. Il me semble que la procédure souhaitable serait la suivante: En cas de violation de la Convention de Genève, l'Etat lésé s'adresserait à la puissance protectrice, qui, après un examen sommaire, communiquerait la réclamation à la partie adverse. S'il s'agit d'élucider des questions de fait, la puissance protectrice nommera, d'accord avec la puissance accusée, une commission d'enquête, composée exclusivement de personnes appartenant à des pays neutres. En effet, il importe que la commission soit composée de personnes qui puissent se transporter indifféremment dans l'un et l'autre des pays belligérants, puisque l'enquête devra souvent se faire des deux côtés.

Peut-être objectera-t-on à ce système qu'il peut conduire à la formation de deux commissions d'enquête, l'une constituée à la demande de l'Etat $A$ qui allègue une violation de la Convention de la part de l'Etat $B$, et l'autre constituée à la demande de l'Etat $B$ qui accuse l'Etat A d'une infraction. A mon avis, la coexistence de ces deux commissions n'offre aucun inconvénient. Bien plus, il serait même difficile à une seule commission de 


\section{Revision de l'article 30 \\ de la Convention de Genève.}

faire simultanément des enquêtes relatives à plusieurs infractions. L'existence de deux commissions faciliterait beaucoup les enquêtes. Du reste, il n'est pas impossible que les deux commissions soient composées des mêmes personnes ; et en tout cas, les deux puissances protectrices (s'il y en a deux), ou les commissions elles-mêmes veilleront à ce que les enquêtes soient coordonnées, s'il y a lieu.

Il est peut-être utile de prévoir expressément le cas d'une violation de la Convention par un belligérant au préjudice d'un neutre (par exemple : un bombardement d'une ambulance neutre). L'Etat neutre doit être autorisé à demander l'ouverture d'une enquête, dans les mêmes conditions que la puissance protectrice, à moins qu'en l'espèce cette dernière ne l'ait déjà requise. Les Etats en cause devraient s'engager à offrir à une commission d'enquête, constituée à la demande d'un neutre, les mêmes facilités qu'à celle qui serait nommée à la demande d'un belligérant. S'il existe un traité de conciliation et d'arbitrage entre le neutre et le belligérant en cause, il faudrait laisser au neutre le choix entre la procédure de ce traité et la procédure prescrite par le nouvel article 30 de la Convention de Genève. En général, la procédure prévue par la Convention de Genève sera plus rapide, mais il peut $\mathrm{y}$ avoir des situations où l'application $d u$ traité de conciliation et d'arbitrage serait plus efficace. En tout cas, la procédure instituée par la Convention de Genève ne saurait exclure postérieurement une autre procédure, prévue par un traité.

Pour compléter le système suggéré ici, il faudrait prévoir un moyen de prévenir les difficultés provenant d'une mésentente entre la Puissance protectrice et le belligérant quant à la composition de la commission. La Convention pourrait prévoir que si la nomination des membres de la commission n'intervient pas dans les 5 jours, dès le dépôt de la demande, la puis- 


\section{Dietrich Schindler.}

sance protectrice s'adressera au Comité international de la Croix-Rouge (ou au corps diplomatique accrédité auprès de l'Etat accusé de violation de la Convention), qui décidera définitivement. L'appel au Comité international de la Croix-Rouge devrait être aussi prévu dans le cas où il n'y a pas de puissance protectrice: l'Etat qui se prétend lésé s'adressera alors directement au Comité international de la Croix-Rouge. Quant aux détails de la procédure, les clauses des traités de conciliation, en particulier le chapitre I de " L'Acte général » de 1928 fournissent des indications utiles. Au reste, il paraît superflu de les préciser avant qu'intervienne une décision sur la constitution d'un organe chargé des enquêtes.

\section{Projet d'un nouvel article 30 de la Convention de Genève de 1929, proposé par M. D. Schindler.}

Article 30.

1. A la demande d'un belligérant, une enquête devra être ouverte au sujet de toute violation alléguée de la Convention. Une fois la violation constatée, les belligérants $\mathrm{y}$ mettront fin le plus promptement possible.

2. L'enquête sera confiée à une Commission composée de trois ou cinq membres choisis parmi les ressortissants de pays neutres. Cette Commission sera nommée par la Puissance protectrice de l'Etat qui allègue la violation, d'accord avec l'Etat incriminé.

3. Si cet accord n'est pas obtenu dans un délai de cinq jours à partir de la demande d'enquête, le Comité international de la CroixRouge constituera lui-même la Commission, sur demande de la Puissance protectrice de l'Etat qui allègue la violation.

4. Le Comité international de la Croix-Rouge constituera également la Commission, sur demande de l'Etat qui allègue la violation, au cas où il n'y aurait pas de Puissance protectrice.

5. Les Puissances protectrices pourront proposer aux belligérants la nomination de Commissions dès le début des hostilités. 


\section{Projet d'un nouvel article 30 de la Convention de Genève.}

6. Sauf accord contraire, la procédure suivie pour l'enquête sera celle qui est prévue au titre III de la Convention de la Haye du 18 octobre 1907 pour le règlement pacifique des conflits internationaux (art. 18 à 36).

\section{Article 30 bis.}

1. Une Puissance neutre peut demander qu'une enquête soit faite au sujet d'une violation de la Convention commise à son égard.

2. La Commission d'enquête sera nommée d'accord entre les parties parmi les ressortissants de Puissances tierces. A défaut d'accord dans les cinq jours à partir de la demande, elle sera nommée, sur requête de l'Etat neutre, par le Comité international de la Croix-Rouge.

3. Les dispositions d'autres traités, notamment en matière d'arbitrage, demeurent en vigueur pour les Etats qui y sont parties. 